\title{
Exigências térmicas de Brevicoryne brassicae e previsão de picos populacionais ${ }^{(1)}$
}

\author{
Francisco Jorge Cividanes ${ }^{(2)}$
}

\begin{abstract}
Resumo - O objetivo deste trabalho foi determinar as exigências térmicas do pulgão-da-couve, Brevicoryne brassicae L., Hemiptera: Aphididae, em condições de laboratório e campo, e prever a ocorrência de picos populacionais de adultos, por meio de um modelo de graus-dia. Em laboratório, os experimentos foram conduzidos em câmaras climatizadas reguladas a $15,20,25,27$ e $30^{\circ} \mathrm{C}$, obtendo-se o limite térmico inferior de desenvolvimento $\left(\mathrm{Tb}=4,5^{\circ} \mathrm{C}\right)$ e a constante térmica $(\mathrm{K}=176,1$ graus-dia). $\mathrm{Na}$ determinação das exigências térmicas sob condições de campo, realizaram-se seis experimentos, a partir dos quais obteve-se o tempo médio de desenvolvimento de ninfas e a temperatura média ambiental. Os resultados estimados de $\mathrm{Tb}$ e $\mathrm{K}$ foram $3,5^{\circ} \mathrm{C}$ e 180,0 graus-dia, respectivamente. Os resultados de $\mathrm{Tb}$ e $\mathrm{K}$ obtidos em laboratório possibilitam prever com maior precisão a ocorrência de adultos de B. brassicae do que os resultados de $\mathrm{Tb}$ e $\mathrm{K}$ determinados a partir de experimentos em campo.
\end{abstract}

Termos para indexação: Brassica oleracea, inseto, pulgão-da-couve, temperatura, modelo.

\section{Thermal requirements of Brevicoryne brassicae and prevision of populational peaks}

\begin{abstract}
The objective of this work was to determine the thermal requirements of Brevicoryne brassicae L., Hemiptera: Aphididae under laboratory and field conditions and to predict the occurrence of peak population of adults, using a degree-day model. In laboratory, the experiments were conducted in climatic chambers at $15,20,25,27$ and $30^{\circ} \mathrm{C}$, and the lower developmental thermal threshold $\left(\mathrm{Tb}=4.5^{\circ} \mathrm{C}\right)$ and the thermal constant $(\mathrm{K}=176.1$ degree-day $)$ were evaluated. The thermal requirements under field conditions were estimated in six experiments, to establish the average time of development of nymphs and the average environmental temperature. The results estimated for $\mathrm{Tb}$ and $\mathrm{K}$ were $3.5^{\circ} \mathrm{C}$ and 180.0 degree-day, respectively. The results of $\mathrm{Tb}$ and $\mathrm{K}$ determined from laboratory data allow more accurate predictions of occurrence of $B$. brassicae adults than the results of $\mathrm{Tb}$ and $\mathrm{K}$ determined from field experiments.
\end{abstract}

Index terms: Brassica oleracea, insect, cabbage aphid, temperature, models.

\section{Introdução}

O pulgão-da-couve, Brevicoryne brassicae (Linnaeus, 1758), se distribui nas regiões temperadas e subtropicais do mundo, causando danos em muitas espécies de brássicas (Ellis \& Singh, 1993). No Brasil, é considerado como uma das pragas mais prejudiciais a essas hortaliças (Longhini \& Busoli, 1993).

Do mesmo modo que ocorre em outros grupos de insetos, o desenvolvimento dos pulgões depende

\footnotetext{
(1) Aceito para publicação em 19 de fevereiro de 2003.

(2) Universidade Estadual Paulista, Faculdade de Ciências Agrárias e Veterinárias, Dep. de Fitossanidade, Via de Acesso Prof. Paulo Donato Castellane, s/n, CEP 14884-900 Jaboticabal, SP. Bolsista do CNPq. E-mail: fjcivida@fcav.unesp.br
}

da temperatura (Campbell et al., 1974; Ro et al., 1998), e dois parâmetros estão relacionados com o efeito da temperatura sobre esses organismos: os limites térmicos superior e inferior de desenvolvimento. Esses dois limites mostram que os pulgões se desenvolvem dentro de uma estreita faixa de variação de temperatura (Higley et al., 1986; Lamb, 1992).

A relação entre velocidade de desenvolvimento dos pulgões e a temperatura apresenta um padrão consistente (Campbell et al., 1974), mostrando-se linear no intervalo de temperatura que abrange os limites térmicos superior e inferior. Portanto, as temperaturas situadas nesse intervalo são empregadas na estimativa dos limites térmicos e da quantidade de graus-dia necessária para a espécie completar seu ciclo biológico (Lamb, 1992). 
Vários autores (Hughes, 1963; Daiber, 1970; Raworth, 1984) determinaram as exigências térmicas de B. brassicae em outros países. No entanto, não existem informações similares sobre esse pulgão no Brasil. Tais estudos têm grande aplicação na entomologia agrícola, e são úteis para o entendimento da ecologia de insetos relacionada com a distribuição e abundância populacional, zoneamento ecológico, tabelas de vida e controle biológico (Messenger, 1970; Parra, 1985; Ro et al., 1998; Cividanes, 2000).

Os resultados de exigências térmicas podem também ser utilizados com modelos que processam a contagem do tempo em graus-dia na previsão do desenvolvimento de pulgões e de outros insetos (Higley et al., 1986; Ro et al., 1998). Cabe ressaltar que modelos de graus-dia têm sido úteis na previsão de ocorrência de adultos de insetos, possibilitando a indicação mais precisa da época de controle ou de amostragem (Wilson \& Barnett, 1983), na construção de modelos de simulação por computador (Higley et al., 1986) e no entendimento de interações ecológicas de insetos-praga e de inimigos naturais (Raffa et al., 1992; Thireau \& Regniere, 1995).

O objetivo deste trabalho foi determinar em laboratório e campo as exigências térmicas de $B$. brassicae, e prever a ocorrência de picos populacionais de adultos em campo na couve, por meio de um modelo de graus-dia.

\section{Material e Métodos}

Os experimentos foram realizados no laboratório de Ecologia de Insetos e no campo experimental do Departamento de Fitossanidade, da Faculdade de Ciências Agrárias e Veterinárias (FCAV), Universidade Estadual Paulista (Unesp), Campus de Jaboticabal, SP, durante o período de julho de 1993 a janeiro de 1994.

Nos experimentos em laboratório, a planta hospedeira utilizada foi o repolho (Brassica oleracea L. var. capitata), híbrido Kenzan Ky, com 90 a 110 dias de idade, plantadas isoladamente em vasos de plástico de $11,5 \mathrm{~cm}$ de altura $\mathrm{x}$ $14,5 \mathrm{~cm}$ de diâmetro superior, tendo como substrato uma mistura constituída de duas partes de solo Latossolo Vermelho-Escuro e uma parte de esterco de curral curtido.

As gaiolas utilizadas para conter os pulgões foram feitas de plástico transparente, em formato circular de $3 \mathrm{~cm}$ de diâmetro x $1 \mathrm{~cm}$ de altura, tendo a superfície superior coberta com voile branco e a inferior tendo apenas a borda coberta por espuma de $3 \mathrm{~mm}$ de espessura. As gaiolas foram fixadas nas folhas das plantas por meio de um prendedor de alumínio, com uma das hastes presa no voile e a outra em um anel de plástico com diâmetro igual ao lado inferior da gaiola.
O tempo de duração da fase ninfal foi determinado em câmaras climatizadas, reguladas a $15,20,25,27$ e $30 \pm 1^{\circ} \mathrm{C}$, 14 horas de fotófase e $70 \pm 10 \%$ de umidade relativa. Para a obtenção de progênies, entre 9 e 11 horas, cerca de quatro a cinco pulgões adultos foram colocados no interior de gaiolas, fixadas em folhas de plantas de repolho, mantidas no interior das câmaras climatizadas. Após sete horas, os adultos foram retirados e 30 ninfas de primeiro ínstar foram individualizadas em gaiolas fixadas nas plantas de repolho, sendo observadas diariamente até se transformarem em adultos. $\mathrm{O}$ delineamento experimental foi inteiramente casualizado, e os resultados foram submetidos à análise de variância, com médias comparadas pelo teste de Tukey a $5 \%$ de probabilidade.

Nos experimentos em campo, o local de estudo situava-se num Latossolo Vermelho-Escuro. Plantaram-se em três áreas de $5 \times 30 \mathrm{~m}$ cada, 180 plantas de repolho em espaçamento de $1 \times 0,5 \mathrm{~m}$. Aplicaram-se $50 \mathrm{~g} /$ cova da fórmula 4-30-10 além de $2 \mathrm{~g}$ de sulfato de boro. Quando necessária, foi efetuada irrigação por aspersão.

O tempo de duração da fase ninfal foi determinado em seis períodos diferentes iniciados em 17, 19 e 30 de julho, 3 de agosto, 9 de outubro e 3 de dezembro de 1993. Para se obter as progênies e acompanhar o desenvolvimento das ninfas, empregou-se o mesmo procedimento e tipo de gaiola dos experimentos realizados em laboratório. Foram mantidas de quatro a seis ninfas de primeiro ínstar por gaiola, totalizando cerca de 150 ninfas observadas por experimento. $\mathrm{O}$ cálculo do tempo médio de desenvolvimento da fase ninfal foi efetuado a partir da data do surgimento das ninfas de primeiro ínstar até a data em que ocorreram $50 \%$ dos adultos.

A temperatura média ambiental foi calculada utilizando-se as temperaturas registradas a partir do momento do aparecimento das primeiras ninfas de primeiro ínstar, até a metade do dia em que ocorreram cerca de $50 \%$ dos adultos, de acordo com o método de Nowierski et al. (1983). A temperatura foi registrada por um termoigrógrafo mantido a $1 \mathrm{~m}$ de altura do solo dentro de um abrigo termométrico, localizado na área do experimento. Esse abrigo foi construído com uma estrutura metálica de $50 \times 50 \times 60 \mathrm{~cm}$ revestida por ripas de madeira pintadas de branco, de acordo com modelo desenvolvido pelo setor de Agrometeorologia do Departamento de Ciências Exatas da FCAV/Unesp.

As exigências térmicas de $B$. brassicae foram determinadas pelo método da hipérbole, calculando-se o limite térmico inferior do desenvolvimento (Tb) e a constante térmica (K), fazendo a regressão linear entre as velocidades do desenvolvimento e as correspondentes temperaturas constantes consideradas no estudo em laboratório (Haddad et al., 1999), ou as médias das temperaturas registradas nos experimentos em campo (Nowierski et al., 1983). 
A previsão de ocorrência de picos populacionais de adultos foi realizada segundo o modelo de graus-dia empregado por Cividanes (2000), que calcula o número de graus-dia por meio do limite térmico inferior do desenvolvimento do inseto e as temperaturas máxima e mínima diárias. As temperaturas utilizadas nesse estudo foram registradas na Estação Agroclimatológica da FCAV/Unesp. A precisão do modelo de graus-dia empregado foi estimada de duas maneiras: a) comparando-se os resultados da previsão do modelo com o tempo do desenvolvimento da fase ninfal nos experimentos em campo, que foi contado em dias, desde o surgimento das ninfas de primeiro ínstar até o dia em que $50 \%$ atingiram a fase adulta; b) estimandose a porcentagem do erro entre a ocorrência de adultos prevista pelo modelo e a observada no campo (West \& Laing, 1984). O erro foi estimado pela fórmula: $\mathrm{E}=100(\mathrm{GDA}-\mathrm{K}) / \mathrm{K}$,

onde GDA: graus-dia acumulados e K: constante térmica de B. brassicae.

\section{Resultados e Discussão}

Nos experimentos em laboratório, a duração da fase ninfal de $B$. brassicae diminuiu significativamente nas temperaturas de 15 a $25^{\circ} \mathrm{C}$, tendendo a estabilizar-se a partir de $25^{\circ} \mathrm{C}$ (Tabela 1). $\mathrm{O}$ aumento observado na duração da fase ninfal entre as temperaturas de $27^{\circ} \mathrm{C}(7,80 \pm 0,28)$ e de $30^{\circ} \mathrm{C}(8,60 \pm 0,31)$, apesar de não-significativo, evidencia que a velocidade de desenvolvimento de $B$. brassicae diminuiu a $30^{\circ} \mathrm{C}$ (Tabela 1, Figura 1). De acordo com Wilson \& Barnett (1983), considera-se o limite térmico superior de desenvolvimento de um inseto a temperatura na qual a velocidade do seu desenvolvimento começa a diminuir. Assim, o limite térmico superior de $B$. brassicae está na faixa de temperatura de $27^{\circ} \mathrm{C}$ até $30^{\circ} \mathrm{C}$. Outros estudos comprovam essas evidências. Daiber (1970) sugeriu $30^{\circ} \mathrm{C}$ como o limite térmico superior do desenvolvimento de B. brassicae estudado em repolho e sob condições de casa de vegetação. Lamb (1961) mencionou que $B$. brassicae apresentou aumento exponencial no consumo de oxigênio com o aumento da temperatura de $8^{\circ} \mathrm{C}$ até $30^{\circ} \mathrm{C}$. Acima de $30^{\circ} \mathrm{C}$, a taxa de respiração diminuiu rapidamente, indicando que alterações metabólicas adversas ocorreram acima dessa temperatura.

Nos experimentos em campo, a duração da fase ninfal de $B$. brassicae mostrou-se semelhante quando obtida durante as condições de inverno (julho e agosto), diminuindo nas temperaturas mais elevadas $\left(22,1^{\circ} \mathrm{C}\right.$ e $\left.27,4^{\circ} \mathrm{C}\right)$, as quais correspondem às médias das temperaturas registradas nos experimentos desenvolvidos no início e final da primavera, respectivamente (Tabela 1 ).

Sob condições de campo, o tempo de desenvolvimento de pulgões e de outros insetos pode ser adversamente afetado por fatores climáticos, nutricionais da planta hospedeira e comportamentais do inseto (Nowierski et al., 1983; Raworth et al., 1984; Higley et al., 1986; Morimoto et al., 1998; Ro et al., 1998), sendo necessária precaução para se utilizar resultados de exigências térmicas desses organismos quando determinados com dados de campo.

Observou-se que ocorreu uma relação linear entre a velocidade de desenvolvimento de $B$. brassicae e a temperatura (Figura 1), possibilitando determinar o tempo de desenvolvimento em termos de grausdia acima do limite térmico inferior de desenvolvimento. A temperatura de $30^{\circ} \mathrm{C}$, utilizada em laboratório, não foi incluída no cálculo das exigências térmicas pois, como discutido anteriormente, prolongou o tempo de desenvolvimento de $B$. brassicae (Figura 1), fazendo com que a velocidade de desenvolvimento alterasse a relação linear proporcionada pelas demais temperaturas. Campbell et al. (1974) recomendam rejeitar o valor da velocidade de desenvolvimento obtido na temperatura mais elevada de um experimento, quando não se ajustar à relação linear observada entre os demais valores.

O limite térmico inferior do desenvolvimento $(\mathrm{Tb})$ encontrado para B. brassicae em condições de laboratório (Tabela 2) aproximou-se do valor de $5,0^{\circ} \mathrm{C}$, obtido por Hughes (1963) em Canberra, Austrália, sendo menor que o resultado da $\mathrm{Tb}\left(6,7^{\circ} \mathrm{C}\right)$ obtido por Raworth (1984) em Vancouver, Canadá. Por outro

Tabela 1. Duração da fase ninfal de Brevicoryne brassicae sob condições de laboratório e de campo. Jaboticabal, SP, 1993/1994(1)

\begin{tabular}{ccccc}
\hline \multicolumn{2}{c}{ Laboratório } & & \multicolumn{2}{c}{ Campo } \\
\cline { 1 - 3 } $\begin{array}{c}\text { Temperatura } \\
\left({ }^{\circ} \mathrm{C}\right)\end{array}$ & $\begin{array}{c}\text { Duração } \pm \mathrm{EP}^{(2)} \\
(\text { dias })\end{array}$ & & $\begin{array}{c}\text { Temperatura } \\
\text { média }\left({ }^{\circ} \mathrm{C}\right)\end{array}$ & $\begin{array}{c}\text { Duração } \pm \mathrm{EP} \\
\text { (dias) }\end{array}$ \\
\hline 15 & $17,0 \pm 0,29 \mathrm{a}$ & 18,2 & $11,9 \pm 0,12$ \\
20 & $11,1 \pm 0,29 \mathrm{~b}$ & 18,5 & $11,9 \pm 0,11$ \\
25 & $8,7 \pm 0,27 \mathrm{c}$ & 19,4 & $11,6 \pm 0,12$ \\
27 & $7,8 \pm 0,28 \mathrm{c}$ & 19,7 & $11,5 \pm 0,11$ \\
30 & $8,6 \pm 0,31 \mathrm{c}$ & 22,1 & $9,4 \pm 0,13$ \\
& & 27,4 & $7,6 \pm 0,14$ \\
\hline
\end{tabular}

${ }^{(1)}$ Médias seguidas pela mesma letra não diferem entre si, pelo teste de Tukey a $5 \%$ de probabilidade. ${ }^{(2)}$ Erro-padrão da média. 
lado, a constante térmica encontrada (Tabela 2) mostrou-se maior que os resultados determinados por Hughes (1963) e Raworth (1984),127 e 126 graus-dia, respectivamente. Não existem referências sobre exigências térmicas de $B$. brassicae determinadas sob condições de campo. Daiber (1970), estudando B. brassicae em casa de vegetação em Pretoria, África do Sul, obteve Tb igual a $5,8^{\circ} \mathrm{C}$ e $\mathrm{K}$ igual a 152 graus-dia, que diferem dos encontrados neste estudo sob condições de campo e de laboratório (Tabela 2).

Campbell et al. (1974), ao relatarem que os resultados das exigências térmicas de uma espécie de pulgão pode variar de uma região para outra, sugeriram que tal característica pode ser um indicativo da adaptação do pulgão às condições climáticas existentes na região. No presente estudo, o baixo limite térmico inferior de desenvolvimento $(\mathrm{Tb})$ encontrado para B. brassicae (Tabela 2) evidencia que essa espécie de pulgão está adaptada a condições climáticas em que predominam temperaturas amenas. Tal evidência corrobora as observações de Daiber (1970),

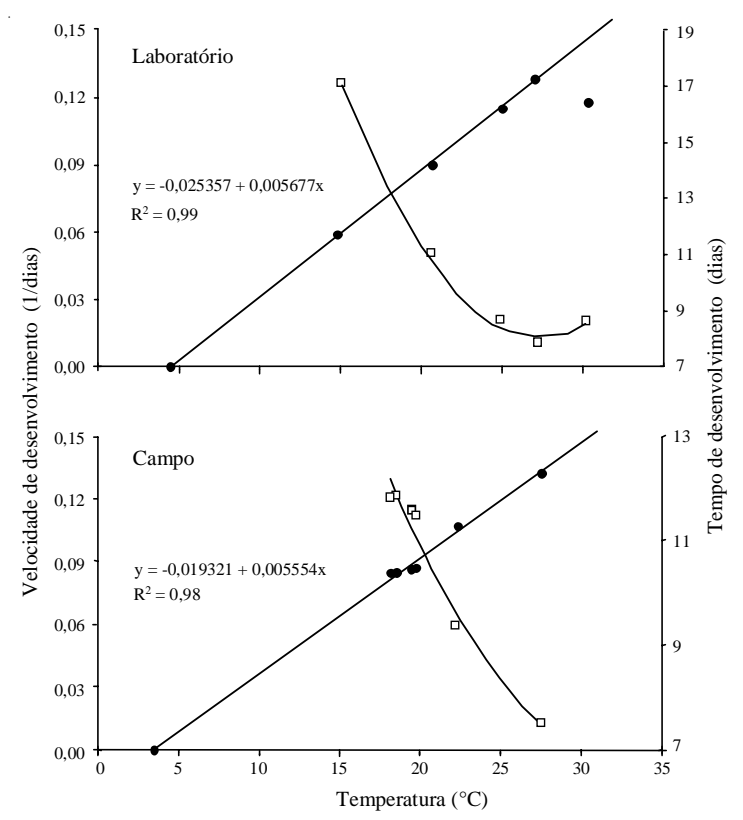

Figura 1. Relação entre a temperatura, o tempo ( $\square$ ) e a velocidade (O) do desenvolvimento de ninfa a adulto de Brevicoryne brassicae em laboratório e em campo. Jaboticabal, SP, 1993/1994. que estudando $B$. brassicae na África do Sul, salientou que essa espécie provoca mais danos em culturas presentes em regiões de temperaturas amenas. Embora B. brassicae esteja amplamente disseminado em países com clima subtropical e temperado, nos países de clima caracteristicamente frio, como a Finlândia e Holanda, o pulgão é considerado a praga mais importante (Markkula, 1953, citado por Daiber, 1970; Hafez, 1961).

Vários trabalhos desenvolvidos no Brasil evidenciam que $B$. brassicae está adaptada a condições de temperaturas amenas. Carvalho et al. (2002) constataram que a maior incidência de formas aladas do pulgão ocorreu durante julho em Lavras, MG. Cividanes (2002a, 2002b) verificou que formas ápteras começaram a infestar a couve em julho, atingindo pico populacional em setembro em Jaboticabal, SP. O autor também constatou que B. brassicae apresentou maior sobrevivência nas condições térmicas de outono e inverno, e que as elevadas temperaturas registradas no verão influenciaram adversamente a dinâmica populacional do pulgão, pois afetaram negativamente seu desenvolvimento, sobrevivência, longevidade e reprodução.

As previsões de ocorrência de adultos pelo modelo de graus-dia foram mais precisas ao utilizar-se os resultados das exigências térmicas obtidos em laboratório, que possibilitaram maior número de previsões coincidentes com a data de ocorrência de adultos de B. brassicae no campo e menores porcentagens de erros que as previsões obtidas com os resultados das exigências térmicas determinados com dados de campo (Tabela 3). Higley et al. (1986) sugeriram que um modelo de graus-dia que proporcione estimativas de previsão com $10 \%$ a $15 \%$ de precisão pode ser adequado para programas de manejo de pragas, sendo necessário que ele apresente precisão um pouco maior para poder vir a ser utilizado em estudos de dinâmica populacional. Portanto, os re-

Tabela 2. Limite térmico inferior de desenvolvimento $(\mathrm{Tb})$, constante térmica $(K)$ e coeficiente de determinação $\left(R^{2}\right)$ do ciclo biológico de ninfa a adulto de Brevicoryne brassicae obtidos sob condições de laboratório e de campo. Jaboticabal, SP, 1993/1994.

\begin{tabular}{lccc}
\hline Local & $\mathrm{Tb}\left({ }^{\circ} \mathrm{C}\right)$ & $\mathrm{K}(\mathrm{GD})^{(1)}$ & $\mathrm{R}^{2}$ \\
\hline Laboratório & 4,5 & 176,1 & 0,997 \\
Campo & 3,5 & 180,0 & 0,983 \\
\hline
\end{tabular}

${ }^{(1)} \mathrm{GD}$ = graus-dia. 
Tabela 3. Ocorrência de adultos prevista em função das exigências térmicas de Brevicoryne brassicae obtidas em condições de laboratório e campo e do aparecimento de 50\% de adultos observado no campo. Jaboticabal, SP, 1993.

\begin{tabular}{|c|c|c|c|c|c|c|}
\hline \multirow{2}{*}{$\begin{array}{c}\text { Data de início } \\
\text { de acumulação } \\
\text { de graus-dia }\end{array}$} & \multicolumn{3}{|c|}{ Laboratório } & \multicolumn{3}{|c|}{ Campo } \\
\hline & $\begin{array}{c}\text { Prevista } \\
(\mathrm{K}=176,1 \text { graus-dia })^{(1)}\end{array}$ & $\begin{array}{c}\text { Observada } \\
\text { (graus-dia acumulados) }\end{array}$ & $\begin{array}{c}\text { Erro } \\
(\%)\end{array}$ & $\begin{array}{c}\text { Prevista } \\
(\mathrm{K}=176,1 \text { graus-dia })\end{array}$ & $\begin{array}{c}\text { Observada } \\
\text { (graus-dia acumulados) }\end{array}$ & $\begin{array}{l}\text { Erro } \\
(\%)\end{array}$ \\
\hline $17 / 7$ & $28 / 7$ & $28 / 7(192,7)$ & 9,4 & $27 / 7$ & $28 / 7(204,7)$ & 13,7 \\
\hline $19 / 7$ & $29 / 7$ & 29/7 $(177,9)$ & 1,0 & $29 / 7$ & 29/7 (192,5) & 6,9 \\
\hline $30 / 7$ & $10 / 8$ & $11 / 8(189,5)$ & 7,6 & $10 / 8$ & $11 / 8(202,2)$ & 12,3 \\
\hline $3 / 8$ & $14 / 8$ & $14 / 8(190,6)$ & 8,2 & $13 / 8$ & $14 / 8(202,5)$ & 12,5 \\
\hline $9 / 10$ & $18 / 10$ & $19 / 10(201,7)$ & 14,5 & $18 / 10$ & $19 / 10(213,7)$ & 18,7 \\
\hline $3 / 12$ & $10 / 12$ & $11 / 12(197,1)$ & 11,9 & $10 / 12$ & $11 / 12(206,1)$ & 14,5 \\
\hline
\end{tabular}

${ }^{(1)} \mathrm{K}=$ constante térmica.

sultados das exigências térmicas de B. brassicae obtidos em laboratório podem ser utilizados em programas de manejo integrado e em estudos de dinâmica populacional desse pulgão.

\section{Conclusões}

1. O limite térmico inferior de desenvolvimento e a constante térmica do ciclo biológico de ninfa a adulto de $B$. brassicae é de $4,5^{\circ} \mathrm{C}$ e de 176,1 graus-dia, respectivamente.

2. O modelo de graus-dia utilizado é adequado para a previsão de ocorrência de adultos de B. brassicae em condições de campo.

\section{Agradecimentos}

Ao Sr. Jairo Guimarães Figueiredo (FCAV-Unesp, Jaboticabal, SP), pela participação na condução dos experimentos em laboratório e campo.

\section{Referências}

CAMPBELL, A.; FRAZER, B. D.; GILBERT, N.; GUTIERREZ, A. P.; MacKAUER, M. Temperature requirements of some aphids and their parasites. Journal of Applied Ecology, Oxford, v. 11, n. 2, p. 431-438, 1974.

CARVALHO, L. M. de; BUENO, V. H. P.; MARTINEZ, R. P. Levantamento de afídeos alados em plantas hortícolas em Lavras-MG. Ciência e Agrotecnologia, Lavras, v. 26, n. 3, p. 523-532, 2002.

CIVIDANES, F. J. Impacto de inimigos naturais e de fatores meteorológicos sobre uma população de Brevicoryne brassicae (L.) (Hemiptera: Aphididae) em couve. Neotropical Entomology, Londrina, v. 31, n. 2, p. 249-255, 2002a.
CIVIDANES, F. J. Tabelas de vida de fertilidade de Brevicoryne brassicae (L.) (Hemiptera: Aphididae) em condições de campo. Neotropical Entomology, Londrina, v. 31, n. 3, p. 419-427, 2002b.

CIVIDANES, F. J. Uso de graus-dia em entomologia com particular referência ao controle de percevejos pragas da soja. Jaboticabal: Funep, 2000. 31 p.

DAIBER, C. C. Cabbage aphids in South Africa: the influence of temperature on their biology. Phytophylactica, Pretoria, v. 2, n. 3, p. 149-156, 1970.

ELLIS, P. R.; SINGH, R. A review of the host plants of the cabbage aphid, Brevicoryne brassicae (Homoptera, Aphididae). International Organization for Biological and Integrated Control of Noxious Animals and Plants: West Palaeartic Regional Section Bulletin, Darmstadt, v. 16, n. 5, p. 192-201, 1993.

HADDAD, M. L.; PARRA, J. R. P.; MORAES, R. C. B. Métodos para estimar os limites térmicos inferior e superior de desenvolvimento de insetos. Piracicaba: Fealq, 1999. 29 p.

HAFEZ, M. Seasonal fluctuations of population density of the cabbage aphid, Brevicoryne brassicae (L.), in the Netherlands, and the role of its parasite, Aphidius (Diaeretiella) rapae (Curtis). Tijdschrift over Planteziekte, Wageningen, v. 67, p. 445-548, 1961.

HIGLEY, L. G.; PEDIGO, L. P.; OSTLIE, K. R. Degday: a program for calculating degree-days, and assumption behind the degree-day approach. Environmental Entomology, Lanham, v. 15, n. 5, p. 999-1016, 1986.

HUGHES, R. D. Population dynamics of the cabbage aphid, Brevicoryne brassicae (L.). Journal of Animal Ecology, Oxford, v. 32, n. 3, p. 393-424, 1963.

LAMB, K. P. Some effects of fluctuating temperatures on metabolism, development, and rate of population growth 
in the cabbage aphid, Brevicoryne brassicae. Ecology, Washington, v. 42, n. 4, p. 740-745, 1961.

LAMB, R. J. Developmental rate of Acyrthosiphon pisum (Homoptera: Aphididae) at low temperatures: implications for estimating rate parameters for insects. Environmental Entomology, Lanham, v. 21, n. 1, p. 10-19, 1992.

LONGHINI, L. C. S. B.; BUSOLI, A. C. Controle integrado de Brevicoryne brassicae (L., 1758) (Homoptera: Aphididae) e Ascia monuste orseis (Latr., 1819) (Lepidoptera: Pieridae), em couve (Brassica oleraceae var. acephala). Científica, São Paulo, v. 21, n. 2, p. 231-237, 1993.

MESSENGER, P. S. Bioclimatic inputs to biological control and pest management programs. In: RABB, R. L.; GUTHRIE, F. E. (Ed.). Concepts of pest management. Raleigh: North Carolina State University, 1970. p. 84-102.

MORIMOTO, N.; IMURA, O.; KIURA, T. Potential effects of global warming on the occurrence of Japanese pest insects. Applied Entomology and Zoology, Tokyo, v. 33, n. 1 , p. $147-155,1998$.

NOWIERSKI, R. M.; GUTIERREZ, A. P.; YANINEK, J. S. Estimation of thermal thresholds and age-specific life table parameters for the walnut aphid (Homoptera: Aphididae) under field conditions. Environmental Entomology, Lanham, v. 12, n. 3, p. 680-686, 1983.

PARRA, J. R. P. Biologia comparada de Perileucoptera coffeella (Guérin-Mèneville, 1842) (Lepidoptera: Lyonetiidae) visando ao seu zoneamento ecológico no Estado de São Paulo. Revista Brasileira de Entomologia, São Paulo, v. 29, n. 1, p. 45-76, 1985.

RAFFA, K. F.; HALL, D. J.; KEARBY, W.; KATOVICH, S. Seasonal life history of introduced basswood thrips
(Thysanoptera: Thripidae) in Wisconsin, with observations on associated thrips species. Environmental Entomology, Lanham, v. 21, n. 4, p. 771-779, 1992.

RAWORTH, D. A. Population dynamics of the cabbage aphid, Brevicoryne brassicae (Homoptera: Aphididae) at Vancouver, British Columbia - II: development, fecundity, and longevity. Canadian Entomologist, Ottawa, v. 116, n. 6, p. 871-878, 1984.

RAWORTH, D. A.; McFARLANE, S.; GILBERT, N.; FRAZER, B. D. Population dynamics of the cabbage aphid, Brevicoryne brassicae (Homoptera: Aphididae) at Vancouver, British Columbia - III: development, fecundity, and morph determination vs. aphid density and plant quality. Canadian Entomologist, Ottawa, v. 116, n. 6, p. 879-888, 1984.

RO, T. H.; LONG, G. E.; TOBA, H. H. Predicting phenology of green peach aphid (Homoptera: Aphididae) using degree-days. Environmental Entomology, Lanham, v. 27, n. 2, p. 337-343, 1998.

THIREAU, J. C.; REGNIERE, J. Development, reproduction, voltinism and host synchrony of Meteorus trachynotus with its Choristoneura fumiferana and C. rosaceana. Entomologia Experimentalis et Applicata, Dordrecht, v. 76, n. 1, p. 67-82, 1995.

WEST, R. J.; LAING, J. E. Development of the potato stem borer, Hydraecia micacea (Lepidoptera: Noctuidae) in the laboratory and field. Proceedings of the Entomological Society of Ontario, Guelph, v. 115, p. 81-87, 1984.

WILSON, L. T.; BARNETT, W. W. Degree-days: an aid in crop and pest management. California Agriculture, Oakland, v. 37, n. 1/2, p. 4-7, 1983. 